\title{
"Medtep DBT": A Dialectical Behavior Therapy Native App and Web Platform for Borderline Personality Disorder Patients and Their Therapists
}

\author{
Jacob Suñol ${ }^{1}$, Josefa María Panisello ${ }^{2}$, Eudald Castell ${ }^{3, *}$, Pedro Juan Tárraga López ${ }^{4}$, \\ Carme Sánchez ${ }^{5}$, Víctor Pérez ${ }^{6}$ \\ ${ }^{1}$ Department of Product \& Engineering, Medtep Inc, San Francisco, United States \\ ${ }^{2}$ Department of Global Health, Medtep Inc. San Francisco, United States \\ ${ }^{3}$ Department of Health Services Research, FUFOSA Health Foundation, Spain \\ ${ }^{4}$ Family and Community Medicine, EAP 5, University of Castilla-La Mancha, Spain \\ ${ }^{5}$ Department of Borderline Personality Program, Neuropsychiatry and Addictions of \\ Hospital del Mar. Parc de Salut Mar de Barcelona, Spain \\ ${ }^{6}$ Neuropsychiatry Department, Neuropsychiatry and Addictions of Hospital del Mar. Parc de Salut Mar. Barcelona, Spain
}

Copyright $\mathrm{O} 2017$ by authors, all rights reserved. Authors agree that this article remains permanently open access under the terms of the Creative Commons Attribution License 4.0 International License

\begin{abstract}
Objective: The present article is aimed at describing the development process of Medtep DBT, a native app and web platform, based on Dialectical Behavior Therapy (DBT) for Borderline Personality Disorder (BPD) patients and their therapists. Materials and Methods: The development of Medtep DBT involved five steps: Analysis of the current mobile apps related to DBT and BPD. Development of a beta version of Medtep DBT in collaboration with psychiatrists, psychologists and BPD patients in knowledge of DBT. Testing the beta version by other BPD patients and introducing changes based on provided feedback. Content validation of the final version by mental health professionals. Results: Content validity has been granted by psychologist and psychiatrist whose experience with the use of the platform has been satisfactory. Feedback from BPD patients has also been positive. Discussion: Medtep DBT can be very useful for both, BPD patients and psychologists and psychiatrists; besides being built on evidence-based DBT standards, it is reinforced by BPD patients' and mental health professionals' feedback and collaboration. Even though its content validity has been granted, a pilot study is being carried out to assess its effectiveness. Conclusion: Medtep DBT facilitates BPD patients the accessibility to digital therapeutic tools to register all the information related to the therapy in a more patient-friendly manner versus paper. Moreover, therapists can provide personalized care in a timely manner. So far, Medtep DBT provides a patient-therapist approach like no other app available in the market.
\end{abstract}

Keywords Dialectical Behavior Therapy, Borderline Personality Disorder, Mental Health, Mobile Applications, Mhealth

\section{Background}

Borderline Personality Disorder (BPD) is a second axis disorder which belongs to the Personality Disorder's "Group B". It usually starts at the beginning of adulthood and the main symptoms are instability in relationships, disturbance of self-identity, high impulsivity, unstable mood, as well as self-harm and suicidal behaviors among others [1]. Its prevalence is between $0.7 \%$ and $5.9 \%$ within the general population [2-7] and its representation in outpatient clinics is up to $22.6 \%$ [8] being one of the most diagnosed personality disorders $[5,6]$. Personality Disorders imply enormous direct and indirect costs, summing up to $11.120 €$ per patient per year, whereby BPD and obsessive-compulsive disorders highly contribute to this mean cost [8]. Indirect and direct costs solely for BPD patients amount to $16.852 €$, which results in one of the considerably highest economic burdens within patients with personality disorders [9]. These expenses gain even more importance when taking into account that around $6 \%$ of primary care patients are diagnosed with BPD [11]. Medication prescription is the simplest way for the treatment of these patients, but not the most recommended one. According to the Practice Guideline for the Treatment of Patients with Borderline Personality [12] the main treatment for BPD is psychotherapy, which can be complemented with pharmacotherapy in order to deal with specific symptoms, providing always psychiatric management during the course of the treatment [13]. Psychological therapies for BPD patients can be both, 
provided individually or in groups, and in both effectiveness has been proved [14]. These therapist-led group sessions may help patients by learning how to interact with others and how to express themselves effectively [15]. As for types of psychological therapies, many have been used, being Dialectical Behavior Therapy (DBT)[16] the most studied one so far[17]. DBT's first effectiveness was demonstrated in patients with suicidal behaviors [16] and later in patients with BPD [18]. It consists of a multimodal approach combining individual therapy, skills training, a therapist consultation team and phone consultation if needed. Standard DBT addresses the following five functions: (1) increasing behavioral capabilities, (2) improving motivation for skillful behavior (through contingency management and reduction of interfering emotions and cognitions), (3) assuring generalization of gains to the natural environment, (4) structuring the treatment environment so that it reinforces functional rather than dysfunctional behaviors, and (5) enhancing therapist capabilities and motivation to treat patients effectively [19].

Since behavioral health has already started getting inside the Mobile Applications world [20], Mobile Health (mHealth) is a field DBT could benefit from. This becomes even more relevant when observing that smartphone ownership has been estimated between $62.5 \%$ and $72 \%$ in psychiatric population which is a considerably high rate [21, 22]. In addition, the growth of the sector in the last few years is indisputable; while in 2014 there were around 100.000 applications globally available in the Health sections of different digital platforms[23], in 2016 the number has risen to 150.000 approximately[24]. Furthermore, interest in mHealth for Mental Health Conditions and its potential is also increasing [25-28]. There is also a will to use mobile apps for mental health by the psychiatric population[21, 22]; regarding the global population, especially the young, around $30,8 \%$ to $38,8 \%$ were found to be looking for mental health information on the internet[29, 30] and $68 \%$ indicated they would use the internet for mental health support if needed[30]. With regard to healthcare professionals, a study in the UK showed that both, medical doctors and junior doctors, had a high level of use of medical applications and that they supported the development of more medical apps for help in their medical and educational practice [31]. Facing this immense amount of different health-related apps, it emanates the need to seek evidence-based effectiveness [26, 32-36]. This is one of the main problems about DBT mobile apps applied to BPD; only one app has a study assessing its effectiveness [37], however is not available at present in the Apple Store or Google Play. The majority of other mobile applications within these markets can be categorized as informative apps without any functionality. On the other hand, there are other apps that enable users to register their emotions and urges into diary cards and some apps provide the user with a set of useful DBT skills. However, none of them could directly connect patients and healthcare professionals in real time through the app and as previously mentioned, all available apps lacked scientific evidence.

\section{Objective}

Medtep DBT allows BPD patients undergoing therapy to keep track of their daily situations, emotions and use of skills learnt during sessions. The main goal of this Care Plan is to ensure patients successfully complete therapy and help patients reduce their amount of critical episodes. It is also aimed at helping healthcare professionals to easily monitor BPD patients undergoing therapy and to reduce valuable treatment time.

\section{Methods}

\subsection{Medtep DBT's Developer}

Medtep Inc. is a Care Management Platform that facilitates lasting behavioral changes by personalizing validated prevention and treatment plans for chronic and rare diseases, and mental health. It is divided into two sections: Medtep Professionals (for healthcare professionals) and Medtep (for patients).

Clinical protocols from leading experts in the healthcare sector are turned into digital Care Plans that patients can easily understand and engage with, favoring increased adherence and system cost reduction. These digital Care Plans are specifically designed to meet the needs of the users. These are typical data entry systems (e.g., custom protocols or integration with third party tracking devices) and processed data representation systems in the form of dashboards (e.g., graphics, statistics or personalized advice). Medtep DBT is one of these digital Care Plans.

\subsection{Development Process}

Contrary to most BPD apps, Medtep DBT provides the DBT as a solid base that has been developed in collaboration with psychologists (3) and psychiatrists (3); having one of them undergone a unique DBT training. As a consequence, the DBT principles can be recognized within the app. Also a group of BPD diagnosed patients (4), who were receiving DBT, participated in the development process of the app by providing their own experiences and needs. On the base of all this information, a Beta version was released subsequently on January 2016. The same group of patients (4) plus additional patients (4) that started with the assessment later on, tested the Beta version and provided their opinions. Taking into account their responses, changes were implemented in order to complete the final version by April 2016.

\subsection{Privacy and Security Issues}

Although it is highly required, the majority of health 
related apps lack a rigorous privacy policy [38, 39]. In contrast, Medtep's privacy policy has been certified to the highest independent and international privacy standards accepted by the industry. The Medtep infrastructure complies with the COPPA (Children's Online Privacy Protection Act of 1998), audited by TRUSTe, as well as the HIPAA (Health Insurance Portability and Accountability Act). Furthermore, Medtep complies with the EU-U.S. Privacy Shield (former Safe Harbor) with regard to the transfer of personal data from the European Union to the United States while taking into consideration EU data protection requirements.

\subsection{Description of Medtep DBT}

Medtep DBT is an online platform developed for any kind of device such as mobile phones or tablets. In addition, it has a web version. Depending on whether the user is a patient or a healthcare professional, the platform view will be different and the tools and features available might vary as well. At any time, both patients and healthcare professionals have access to a Help Center aimed at giving support and providing tutorials on the main features.

\subsubsection{Patient View - Native App and Web}

Patients can either access the platform directly on their own or be invited by a healthcare professional. They will find three main tools: "Diary Card", "Chain Analysis" and "Crisis plan".

\subsection{2. "Diary Card"}

It gathers all the information related to Urges and Actions, Emotions and Use of Skills on a daily base. The Diary Card usually forms an integral part of DBT. It is very valuable for tracking certain behaviors and helps to determine what to discuss in the next therapy session [15].

"Diary Card: Urges and Actions": This part of the Diary Card registers all the needs and actions the patient faces every day. Committing suicide, performing self-harm, taking drugs, drinking alcohol, and taking extra medication are the main items that the app asks the patient for. However, he can also introduce any other kind of Urges or Actions. Therefore, he assesses by means of a visual scale from 1 to 5 how strong his urge was, whereby 1 means the urge was soft and 5 really strong. In addition, a color scale from green to red is used, whereby green belongs to 1 and red to 5 . Every scale is followed by a legend that explains each number's meaning (Figure 1). As a next step, the patient is asked about the corresponding action. In case he inflicted self-harm, he has to enter the exact action (Figure 2). In case he took drugs or extra medication, or he drank alcohol, the patient is provided a list where he selects the substance with its respective amount and unit to properly indicate his intake.

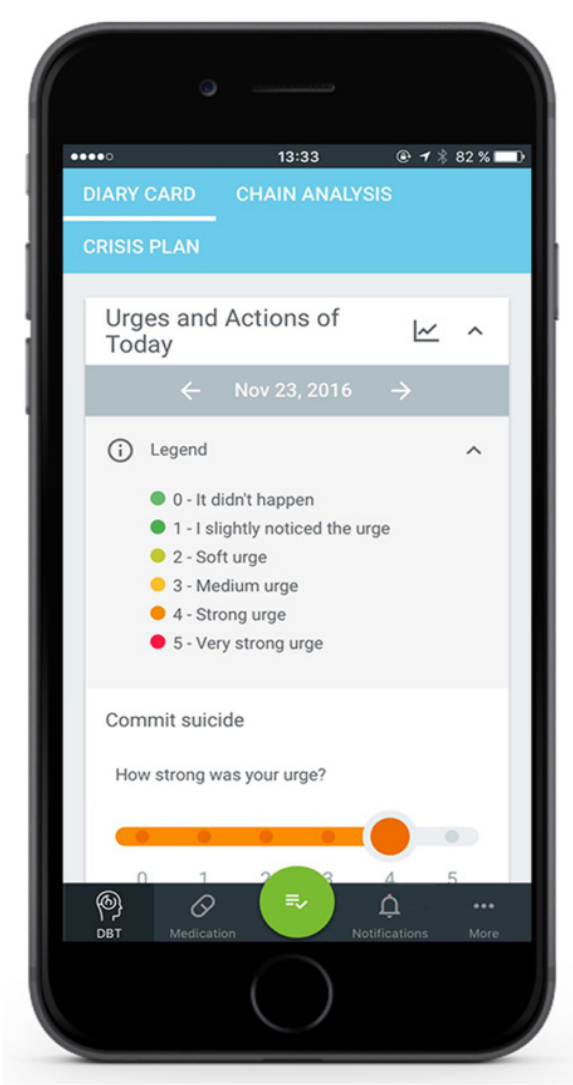

Figure 1. Legend of the items of the Diary Card: Urges and Actions.

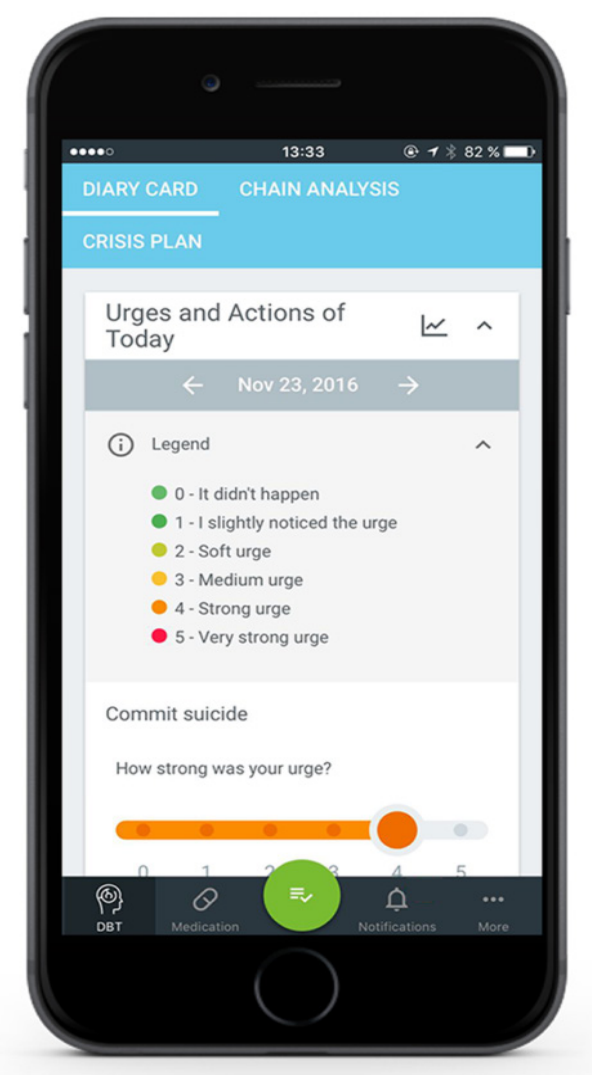

Figure 2. Items of the Diary Card: Urges and Action and its fulfillment. 
"Diary Card: Emotions": The patient can choose one or several emotions from an extensive list or he can introduce them via free text. Once an Emotion is chosen, the patient rates how intense its related feeling was. He chooses again from a scale from 1 to 5 , whereby 1 implies low intensity (light blue) and 5 great intensity (dark blue).

"Diary Card: Use of Skills": First of all, the patient needs to register the Use of Skills. There is a scale from 0 to 8 with different options: 0: Not thought about or used. 1: Thought about, not used, didn't want to. 2: Thought about, not used, wanted to. 3. Tried, but couldn't use them. 4: Tried, could do them, but they didn't help. 5: Tried, could use them, helped. 6: Automatically used them, but didn't help. 7: Automatically used them, helped. These eight options accompany a color scheme, turning from red (0) into green (7). After rating the Use of Skills, the patient chooses the utilized Skills from a list that classifies them into the four basic fields of DBT: Mindfulness, Interpersonal Effectiveness, Emotional Regulation and Distress Tolerance. All skills offer an information icon that provides details about the skills in particular. Reinforcing the registration and Use of Skills, and providing the option to include them into the Crisis Plan results to be very beneficial for BPD patients since increasing the use of skills has been proved to be a mechanism of change for depression, anger control and suicidal behavior[40].

\subsection{3. "Chain Analysis"}

This feature allows a detailed description and evaluation of conflictive situations the patient encounters day-to-day. It consists of seven steps:

1. Vulnerability Factors: The chain analysis' first step requires the patient to enter action/s and issues in his environment that made him feel vulnerable, as well as feelings. If the patient is unsure about its explanation, he can make use of provided suggestions.

2. Prompting Event: This step relates directly to the conflict situation. It requests its exact time as well as a brief description of the situation. There is also the option to easily relate the on-going Chain Analysis with an emotional crisis.

3. Thoughts: At this point, the patient is required to describe the thoughts he had during the event, and beliefs or expectations that followed the event.

4. Physical Sensations: The patient can point out any kind of physical sensation he faced during the event and he can use suggestions within the platform facilitate his expression.

5. Emotions: All the feelings and emotions the patient felt at that situation should be written down and a list of suggestions is again available.

6. Problem Behavior: In the penultimate step the patient is asked two different questions. The first one relates to the action, the patient wanted to realize and if it was a problem behavior. The second one asks how he actually acted, and whether or not he used Skills. In case he did not, this step encourages the patient to write down which Skills could have been useful to overcome the problem. It also offers the possibility of developing a prevention plan to reduce vulnerability to stressful events.

7. Consequences: The last step asks about the consequences of the behavior for the patient and others. In case there were negative consequences, the patient needs to introduce actions that were necessary to handle them.

Finally, the patient has the opportunity to rate how satisfied he was with the Use of Skills during the event that he learned in the therapy. He can choose from 1 to 5 stars, according to an increasing satisfaction. This results in the patient's reinforcement to use the App and the provided Skills.

During the whole Chain Analysis, a scheme at the bottom end of the page indicates the seven steps and marks the phase where the patient is currently in, giving a sensation of continuous progress (Figure 3 ).

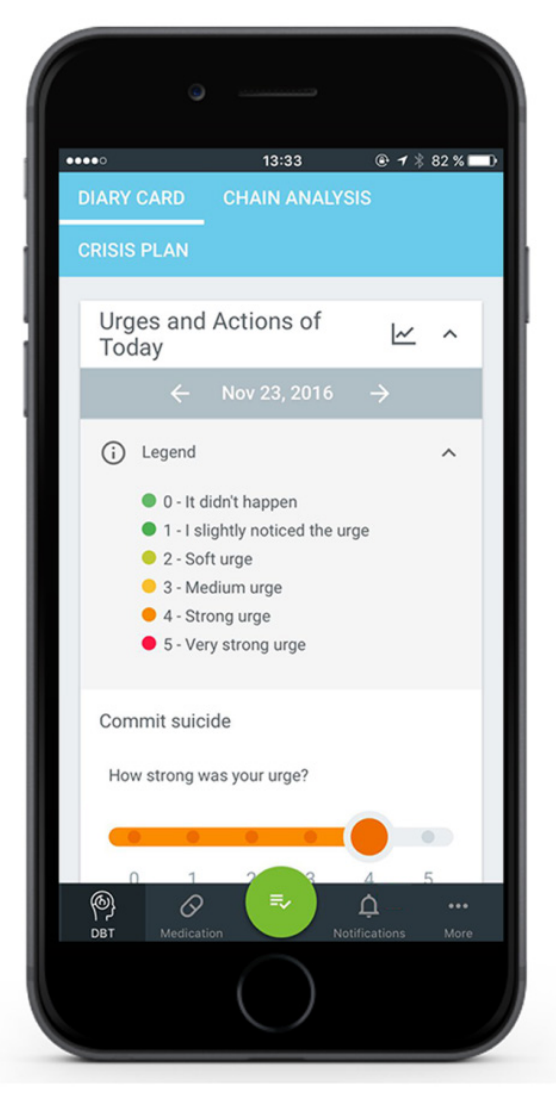

Figure 3. Prompting Event, which is the second step of the Chain Analysis, at the bottom of the screen also appears the small scheme of progression.

\subsection{5. "Crisis Plan"}

This is one of the main features of Medtep DBT, allowing the patient to create and make use of his own Crisis Plan during conflict situations. It gives the patient indications created by himself which increases its effectiveness. This feature is available $24 \mathrm{~h}, 7$ days a week, since a crisis can 
emerge at any time. These indications can either be actions, which work solely for him, or specific DBT skills. The patient can always edit his Crisis Plan, change the order, or eliminate any procedure. Furthermore, he has the option to individualize the Crisis Plan by providing a title and description to every step in order to overcome his crisis more effectively. At the bottom of the plan the patient can find the Emergency Services's number and, if added, also the number of a certain person as his Emergency Contact (Figure 4). Since the situations of crisis normally appear all of a sudden and are the main problem to deal with as a BPD patient, he can always find the exclamation icon at the bottom of any screen in the app in order to quickly access the Crisis Plan. In addition, he has the option to directly insert this icon into the main screen of his electronic device. This feature is of major importance since one of the criteria for diagnosing BPD is suicidal behaviors [1]. Furthermore, it has been discovered that affective instability, which also is a diagnosis criterion and a main characteristic of BPD, is strongly associated with suicidal behaviors [41].

\subsection{6. "Summary"}

After completing the Diary Card, the patient has access to a comprehensive dashboard that collects all the information of past occasions. That gives the patient a complete perspective about his actual state and his progress. Aggregated data is shown on a weekly base together with a weekly average of the Urges, Emotions and Use of Skills ratings. Historical records of Chain Analysis can be accessed at any time.

Moreover, patients have access to their own profile, where they can manage their personal data and settings, such as notifications and sharing permissions with the healthcare professional.

\subsubsection{Professional View - Web}

Medtep Professionals enables therapists to keep a timely follow-up of the patient's progress prior, during and after the individual session or group therapy. They can see each individual patient's dashboard with the registered information for every day, as well as the weekly or monthly information of their whole group. Thereby, they can identify the Urges of doing certain Actions and whether the patient realized them or not. The color scheme complies with the same standards as in the Patient View and whenever an action took place; a small description appears, accordingly. The same system applies to Emotions, and for both the weekly rating average is provided (Figure 5). On top of that, the view of Use of Skills adds a list of the most used skills, giving the healthcare professional information about which strategies suit best for each of his patients. The Chain Analysis view shows a brief description, its creation time and the patient's final rate of satisfaction. However, the therapist can also access every single step of the Chain Analysis. In addition, Medtep DBT allows healthcare professionals to revise the patient's adherence to his daily medication and change it if needed (Figure 6). Another feature allows him to send text messages to the patient directly through the app, for example in case he identifies a dysregulation in the patterns of medication in-take.

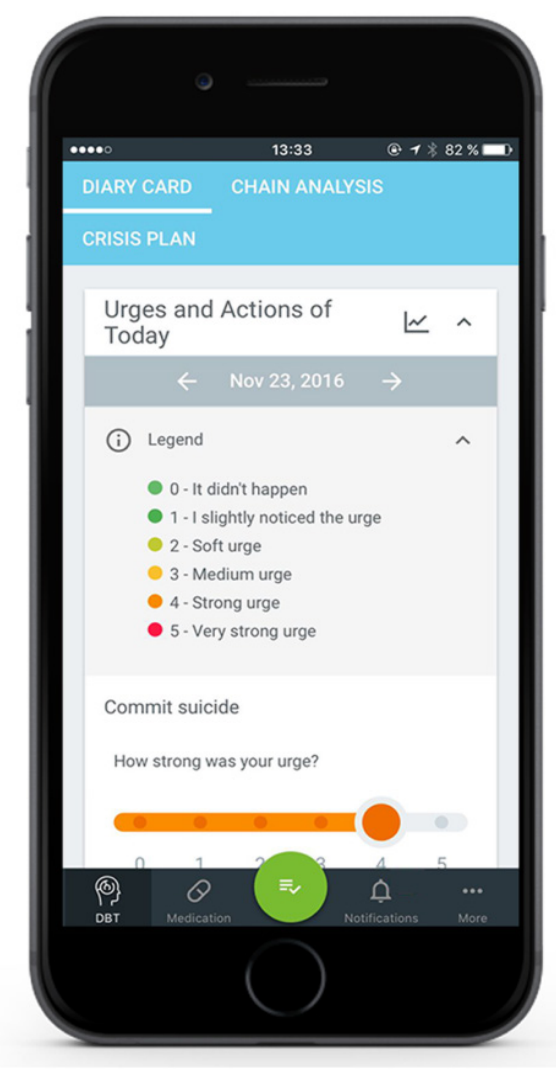

Figure 4. Patient Crisis Plan with the emergency services and the emergency contact at the bottom. 


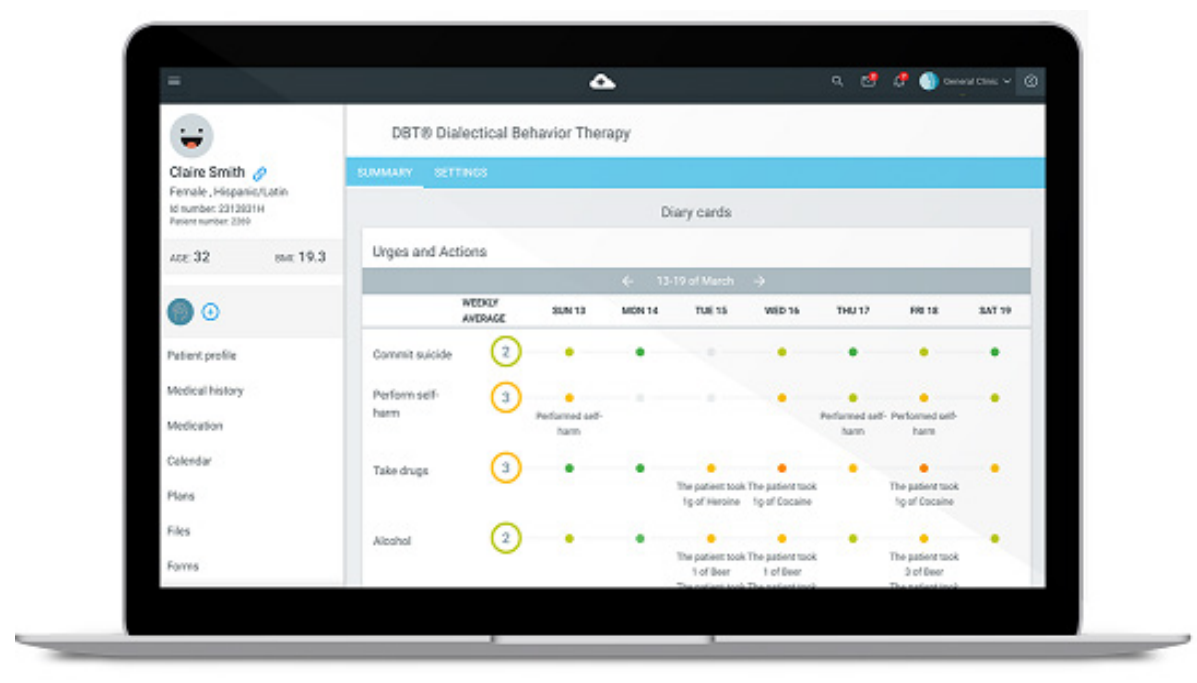

Figure 5. Patient dashboard from the Professional View with all the registered and summarized data from the patient

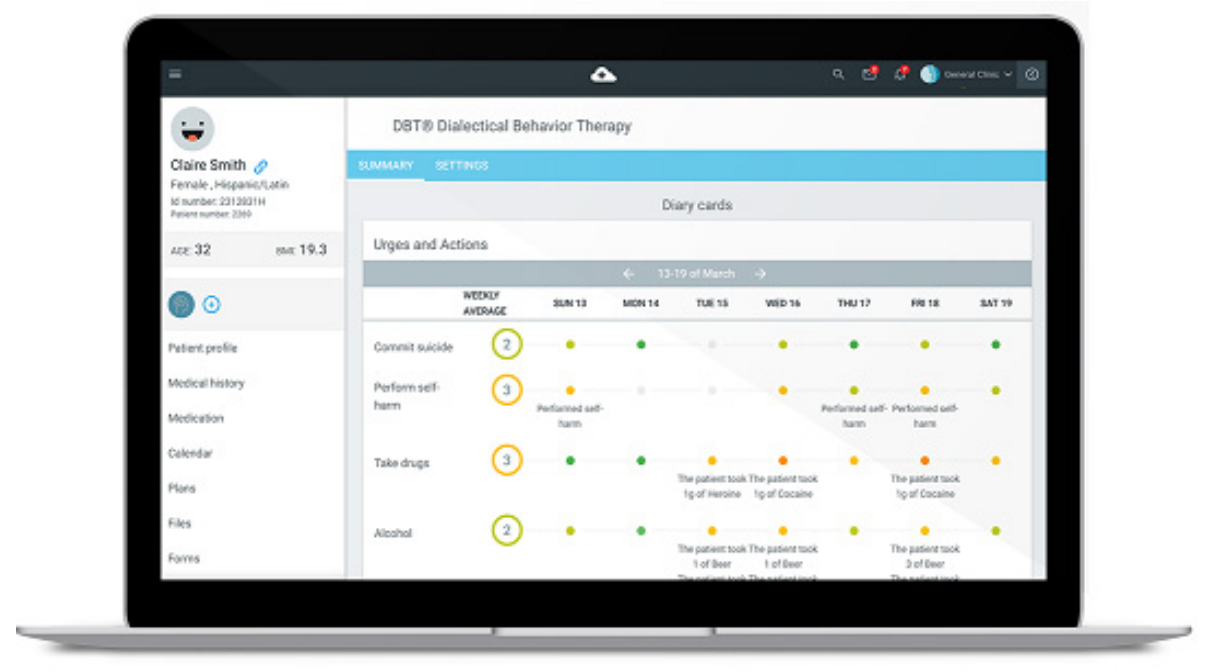

Figure 6. Brief description of the patient Chain Analyses and his register of daily medication in-take from the Professional View

\section{Results}

Expert Testing: Both psychologists and psychiatrists were given Medtep DBT in order to provide content validity. They all tested the platform by having unrestricted access and receiving initial user instructions via email. Based on their professional experience and in-depth knowledge, the experts reported that the app meets successfully the underlying criteria. As a conclusion, Medtep DBT received its content validity.

Potential Users Testing: A group of BPD-diagnosed patients who had never had any contact with the app before (10) formed the group of potential testers and were asked to provide feedback once they had tested the app. The gathered feedback was very satisfying and was taken into account for the final version which received the content validity. Most of the testers reported how useful was for them to be able to register and summarize everything they were feeling or thinking at any time or moment.
"It is awesome to register everything that happens to you whenever you want"

Also several testers reported how understood they felt when they realized that the therapist already knew how they had felt or what had happened to them since the last session.

"It is fantastic to see that your therapist knows already what has happened to you in the last week, it was so much easy to explain myself"

We saw that many of the testers used Medtep DBT as a dictionary that helped them explain their feelings.

"I wasn't able to describe my own feelings until I used the app, the definitions the app provides are clear and there are many"

Pilot: In order to validate the app's effectiveness, a randomized Pilot Study is being carried out. It consists of a pre-post study that assesses whether receiving DBT while 
using Medtep DBT increases its effectiveness compared to receiving DBT while using diary cards in paper. The pilot-study consists of 20 BPD patients and its total duration amounts to 6 months. Behavior and psychological functioning and outcomes with regard to treatment usage are measured. This Pilot Study is executed in collaboration with the Institut de Neuropsiquiatria i Addiccions Parc de Salut MAR.

\section{Discussion}

Medtep DBT is an app and web online platform based on Dialectical Behavior Therapy and created for patients who are diagnosed with BPD and their respective therapists. The app is publicly available in the two biggest markets for apps which are Google Play and Apple Store. Compared to those apps currently found in these markets, Medtep DBT is the only one that: 1. Connects patients and healthcare professionals giving an aggregated view of the collected data through the platform. 2. Allows the healthcare professional to see all data from all of his patients as a summary. 3 . Provides the patient's location through geolocalization in the case of a potential suicidal situation described by the patient; a feature which is only available upon patient's permission. 4 . Gives a quick access to the Crisis Plan in any screen of the app as well as in the device's main screen. 5. Not only provides a validated content but it also carries out a pilot study in order to demonstrate its effectiveness. 6. Allows not only communication between patients and psychotherapists but also with other healthcare professionals. 7. Has been certified to the highest independent and international privacy standards accepted by the industry.

Limitations: The following limitations have been identified and are already being addressed: 1. Despite Medtep DBT's content validity, it might be difficult to use for someone with a lack of knowledge about DBT. 2. At present, it only applies for BPD patients. 3. It does not provide a social feature like forum or chat that would enable the communication between users. 4. Even though there is some reinforcement in the app (e.g. rating the satisfaction from 0 to 5 stars for the use of skills after chain analysis) there is a lack of gamification and there is still room for improvement in this field.

\section{Conclusions}

Medtep DBT includes all the elements from evidence-based DBT; it allows Diary Card (which includes urges and actions, emotions and use of skills) registering, enhances understanding of patient's crisis through the Chain Analysis and enables BPD patients to build and use their own Crisis Plan. Medtep DBT guides patients through the learning process and use of the different therapeutic tools. Reminders and notifications become a great help for patients to keep track of all the information they need to introduce. Information is stored and accessible at any moment in the cloud, making it easier to consult by the therapists and other healthcare professionals in the mid and long term. It provides an aggregated view of patients' progress and detailed individual information, facilitating the analysis and personalized care.

\section{Acknowledgements}

The authors are especially grateful to Dr. Marsha M. Linehan for her permission to develop this project and all Department of product \& engineering team of Medtep Inc. Also recognitions attribute to Parc de Salut MAR. The authors would like to thank the Product Department of Medtep Inc. for their hard work and commitment to the project.

\section{Competing Interests}

Josefa María Panisello is medical director for Medtep Inc and receives a salary and stock options.

Jacob Suñol is cofounder of Medtep Inc and he receives a salary and stock options.

\section{Funding}

This research received no specific grant from any funding agency in the public, commercial, or non-profit sectors.

\section{REFERENCES}

[1] American Psychiatric Association. Diagnostic and statistical manual of mental health disorder $\left(5^{\text {th }}\right.$ ed.). Arlington, VA: American Psychiatric Publishing 2013.

[2] Torgersen S, Kringlen E, Cramer V. The prevalence of personality disorders in a community sample. Arch Gen Psychiatry 2001; 58(6):590-6.

[3] Coid J, Yang M, Tyrer P, et al. Prevalence and correlates of personality disorder in Great Britain. Br J Psychiatry 2006; 188: $423-31$.

[4] Lenzenweger MF, Lane MC, Loranger AW, et al. DSM-IV personality disorders in the National Comorbidity Survey Replication. Biol Psychiatry 2007; 62(6): 553-64.

[5] Grant BF, Chou SP, Goldstein RB, et al. Prevalence, Correlates, Disability, and Comorbidity of DSM-IV Borderline Personality Disorder: results from the Wave 2 National Epidemiologic Survey on Alcohol and Related Conditions. J Clin Psychiatry 2008; 69(4): 533-545.

[6] Rull TJ, Jahng S, Tomko RL, et al. Revised Nesarc Personality Disorder Diagnoses: Gender, Prevalence, and Comorbidity 
with Substance Dependence Disorders. J Pers Disord 2010; 24(4):412-426.

[7] Zanarini MC, Horwood J, Wolke D, et al. Prevalence of DSM-IV borderline personality disorder in two community samples: 6,330 English 11-year-olds and 34,653 American adults. J Pers Disord 2011; 25(5): 607-19.

[8] Korzekwa MI, Dell PF, Links PS, et al. Estimating the prevalence of borderline personality disorder in psychiatric outpatients using a two-phase procedure. Compr Psychiatry 2008; 49(4):380-6.

[9] Soeteman DI, Hakkaart-van Roijen L, Verheul R, et al. The economic burden of personality disorders in mental health care. J Clin Psychiatry 2008; 69(2): 259-65.

[10] Van Asselt ADI, Dirksen CD, Arntz A, et al. The cost of borderline personality disorder: societal cost of illness in BPD-patients. Eur Psychiatry 2007; 22(6): 354-361.

[11] Gross R, Olfson M, Gameroff M, et al. Borderline Personality Disorder in Primary Care. Arch Intern Med 2002; 162: 53-60.

[12] Oldham JM. Guideline Watch: Practice Guideline for the Treatment of Patients with Borderline Personality Disorder. Arlington, VA: American Psychiatric Association 2005.

[13] Chanen AM, Thompson KN. Prescribing and borderline personality disorder. Australian Prescriber 2016; 39 (2):49-53.

[14] Linehan MM, Korslund KE, Harned MS, et al. Dialectical Behavior Therapy for High Suicide Risk in Individuals With Borderline Personality Disorder: A Randomized Clinical Trial and Component Analysis JAMA Psychiatry 2015; 72(5):475-82.

[15] Salsman NL, Linehan MM. Dialectical-Behavior Therapy for Borderline Personality Disorder. Prim psychiatry 2006; 13(5):51-58

[16] Linehan MM. Dialectical Behavioral Therapy: A Cognitive Behavioral Approach to Parasuicide. J Pers Disord 1987; 1(4):328-333.

[17] Stoffers JM, Völlm BA, Rücker G, et al. Psychological therapies for people with borderline personality disorder. Cochrane Database Syst Rev 2012; 15(8).

[18] Linehan MM, Armstrong HE, Suarez A, et al. Cognitive-behavioral treatment of chronically parasuicidal borderline patients. Arch Gen Psychiatry 1991; 48(12): 1060-4.

[19] Linehan MM. Skills training manual for treating borderline personality disorder. Diagnosis and treatment of mental disorders. New York, NY: Guilford Press 1993.

[20] Luxton DD, McCann RA, Bush EN, et al.. mHealth for Mental Health: Integrating Smartphone Technology in Behavioral Healthcare. Prof Psychol Res Pr 2011; 42(6):505-512.

[21] Torous J, Friedman R, Keshavan M. Smartphone Ownership and Interest in Mobile Applications to Monitor Symptoms of Mental Health Conditions. JMIR Mhealth Uhealth 2014;2(1).

[22] Torous J, Chan SR, Tan SY-M, et al. Patient Smartphone Ownership and Interest in Mobile Apps to Monitor Symptoms of Mental Health Conditions: A Survey in Four
Geographically Distinct Psychiatric Clinics. JMIR Ment Health 2014; 1(1).

[23] Research2Guidance. mHealth App Developer Economics 2014: The State of the Art of mHealth App Publishing. [accessed 20/10/2016]

http://research2guidance.com/wp-content/uploads/2015/10/m Health-App-Developer-Economics-2014-Preview.pdf

[24] Research2Guidance. mHealth App Developer Economics 2016: The current status and trends of the mHealth app market. [accessed 20/10/2016] http://research2guidance.com/wp-content/uploads/2016/10/m Health-App-Developer-Economics-2016-v17-Preview-1-1.pd $\mathrm{f}$

[25] Kazdin AE, Blase SL. Rebooting Psychotherapy Research and Practice to Reduce the Burden of Mental Illness. Perspect Psychol Sci 2011; 6(1):21-37.

[26] Price M, Yuen EK, Goetter EM, et al. mHealth: A Mechanism to Deliver More Accessible, More Effective Mental Health Care. Clin Psychol Psychother 2014; 21(5):427-436.

[27] Torous J. Current research and trends in the use of smartphone applications for mood disorders. Internet Interventions 2015; 2(2):169-173.

[28] Leigh S, Flatt S. App-based psychological interventions: friend or foe? BMJ 2015; 18:97-99.

[29] Biddle L, Donovan J, Hawton K, et al. Suicide and the Internet. BMJ 2008; 336: 800 .

[30] Horgan A, Sweeney J. Young students' use of the Internet for mental health information and support. $J$ Psychiatr Ment Health Nurs 2010; 17(2):117-23.

[31] Payne K, Wharrad H, Watts K. Smartphone and medical related Apps use among medical students and junior doctors in the United Kingdom (UK): a regional survey. BMC Med Inform Decis Mak 2012; 12:121.

[32] World Health Organization 2011. mHealth: New horizons for health through mobile technologies: second global survey on eHealth. [accessed 21/10/2016]

http://apps.who.int/iris/bitstream/10665/44607/1/9789241564 250_eng.pdf

[33] Tomlinson M, Rotheram-Borus MJ, Swartz L, et al. Scaling Up mHealth: Where Is the Evidence? PloS Med 2013; 10(2).

[34] Powell AC, Landman AB, Bates DW. In search of a few good apps. JAMA 2014; 311(18):1851-1852.

[35] Olff M. Mobile mental health: a challenging research agenda. Eur J Psychotraumatol 2015; 6.

[36] Marley J, Farooq S. Mobile telephone apps in mental health practice: uses, opportunities and challenges. BJPsych Bull 2015; 39(6):288-290.

[37] Rizvi SL, Dimeff LA, Skutch J, et al. A pilot study of the DBT coach: an interactive mobile phone application for individuals with borderline personality disorder and substance use disorder. Behav Ther 2011; 42(4):589-600.

[38] Sunyaev A, Dehling, T., Taylor PL, et al. Availability and quality of mobile health app privacy policies. $\mathrm{J} \mathrm{Am} \mathrm{Med}$ Inform Assoc 2015; 22:28-33. 
[39] He D, Naveed M, Gunter CA, et al. Security Concerns in Android mHealth Apps. AMIA Annu Symp Proc. 2014; 645-54.

[40] Neacsiu AD, Rizvi SL, Linehan MM. Dialectical behavior therapy skills use as a mediator and outcome of treatment for borderline personality disorder. Behav Res Ther 2010; 48(9):832-9

[41] Yen S, Shea MT, Sanislow CA, et al. Borderline Personality Disorder Criteria Associated With Prospectively Observed Suicidal Behavior. Am J Psychiatry 2004; 161(7); 1296-1298. 